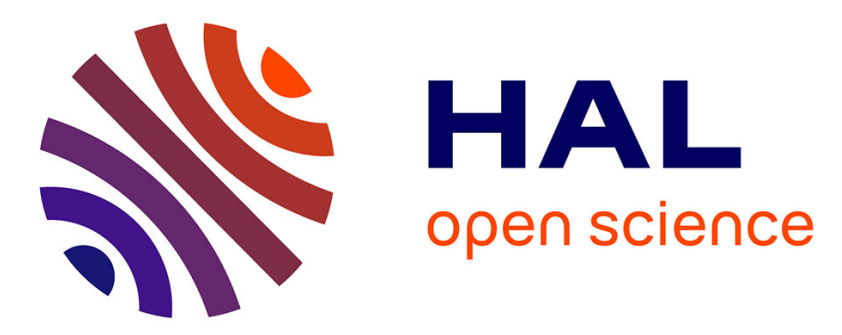

\title{
Possibility of non-invasive blood pressure estimation by measurements of force and arteries diameter
}

Florent Veye, Sandrine Mestre, Antonia Perez-Martin, Jean Triboulet

\section{To cite this version:}

Florent Veye, Sandrine Mestre, Antonia Perez-Martin, Jean Triboulet. Possibility of non-invasive blood pressure estimation by measurements of force and arteries diameter. BHI: Biomedical and Health Informatics, Jun 2014, Valencia, Spain. lirmm-01007425

\section{HAL Id: lirmm-01007425 \\ https://hal-lirmm.ccsd.cnrs.fr/lirmm-01007425}

Submitted on 16 Jun 2014

HAL is a multi-disciplinary open access archive for the deposit and dissemination of scientific research documents, whether they are published or not. The documents may come from teaching and research institutions in France or abroad, or from public or private research centers.
L'archive ouverte pluridisciplinaire HAL, est destinée au dépôt et à la diffusion de documents scientifiques de niveau recherche, publiés ou non, émanant des établissements d'enseignement et de recherche français ou étrangers, des laboratoires publics ou privés. 


\title{
Possibility of non-invasive blood pressure estimation by measurements of force and arteries diameter
}

\author{
Florent VEYE ${ }^{1}$, Sandrine MESTRE ${ }^{2}$, Antonia PEREZ-MARTIN ${ }^{3,4}$, and Jean TRIBOULET ${ }^{1,5}$
}

\begin{abstract}
Ultrasound examination is the first line procedure for the diagnosis and follow-up of cardiovascular diseases. Instrumenting an ultrasound probe with a force sensor may improve thenon-invasive measurement of arterial biomechanical parameters (diameter, pulsatility, intima-media thickness and flow-dependent dilation) by measuring and controlling the force exerted by the sonographer. We present here the results obtained with this approach coupled with image processing for the measurement of arterial diameter changes during the cardiac cycle and dynamic tests. The analysis of artery force feedback provided non-invasively an estimation of blood pressure waveform.
\end{abstract}

\section{INTRODUCTION}

The non-invasive diagnosis and follow-up of cardiovascular diseases need reliable measurements of arterial function. The evaluation of the arterial wall elastic modulus can be based on distensibility measurement as the ratio of arterial diameter and blood pressure changes during the cardiac cycle [1-8]. This assessment can be improved by the simultaneous measurement of the arterial wall thickness and recording of the blood pressure curve at the same site $[9,10]$. Arterial diameter and wall thickness measurements require high resolution ultrasound scanners and dedicated image processing software. B-mode echographic images analysis is generally performed with non-isotropic filtering to smooth speckle noise, and level set methods for contour extraction [11]. Nevertheless, these methods proved not robust enough for this application. Therefore, we used simple but robust operators to extract the arterial wall contour and estimate vessel diameter and diameter changes.

The effect of the pressure exerted by the operator on the ultrasound probe is generally not taken into account during arterial measurements although it is liable to affect the amplitude and the waveform of the diameter changes upon which distensibility measurements are based [12]. The validity of non-invasive arterial pressure measurement (sphygmomanometry, oscillometry...) remains controverted when applied to arteries others than the brachial artery, but is widely used in this context.

\footnotetext{
${ }^{1}$ LIRMM - UMR 5506 - UM2 - CNRS, 161 rue Ada 34095 Montpellier, France.

${ }^{2}$ Internal and Vascular Medicine department - Montpellier University Hospital Center, 191 avenue du Doyen Gaston Giraud 34295 Montpellier, France.

${ }^{3}$ Vascular Medicine and Laboratory department - Nîmes University Hospital Center, Place du Pr R-Debré 30029 Nîmes, France.

${ }^{4}$ Dysfunctions of Vascular Interfaces Laboratory - EA2992 - UM1, Montpellier and Nîmes, France.

${ }^{5}$ Nîmes University, Place Georges Salan 30921 Nîmes, France.
}

Different, and often complex, approaches, have been proposed to estimate the local arterial pressure from the conventional measurement performed on the brachial artery [13]. Most of these approaches are based on a priori estimation of the modifications that undergo the pressure wave during its propagation along arteries, while vascular diseases, by their consequences on the biomechanics of arterial wall, are likely to affect this propagation. Obtaining the arterial blood pressure waveform at the exact site of diameter measurement would drastically improve the accuracy and the reliability of the arterial distensibility measurement.

Our aim was to couple the measurement of the force exerted on the ultrasound probe with image analysis for the evaluation of arterial distensibility in order to obtain an image of the local blood pressure waveform. In the first part of this article, we present the corresponding hardware and software developments. In the second part, we report the clinical results we obtained; showing a correlation between the arterial diameter changes and the force feedback curve. To conclude, we discuss the potential applications of this approach.

\section{MEASUREMENT SYSTEM}

\section{A. Hardware and software developments}

The acquisition system was composed mainly of a computer equipped by a Matrox ${ }^{\circledR}$ analogic video acquisition card and by a National Instruments ${ }^{\circledR}$ multifunction data acquisition board. The video acquisition card was connected to the analogic video output of the ultrasound scanner via a coaxial cable. A force sensor (XFTC300, FPG Sensors, LesClayes-Sous-Bois, France) was connected to a signal amplifier/conditioner (ARD154, FPG Sensors, Les-ClayesSous-Bois, France) whose output was connected to the data acquisition board. The linear force sensor was fixed on a sliding support receiving the linear probe of the ultrasound scanner (Fig.1). Clinical results were obtained with a Logiq 9 duplex Doppler ultrasound system (GE Healthcare Ultrasound, Milwauke, USA) with a $10 \mathrm{MHz}$ linear probe.

The workflow included video and force signal acquisition and synchronized B-mode ultrasonographic image processing for the measurement of arterial diameter and diameter changes. 


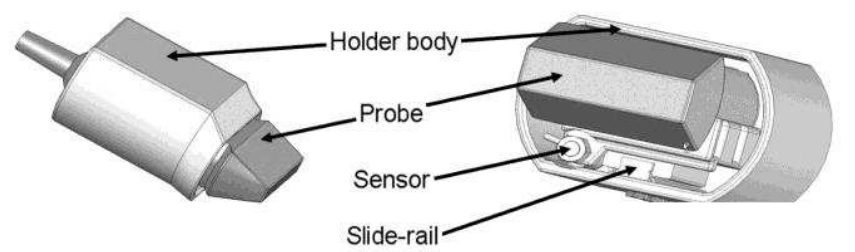

Fig. 1 Drawing showing the linear sensor implementation on the linear ultrasound probe.

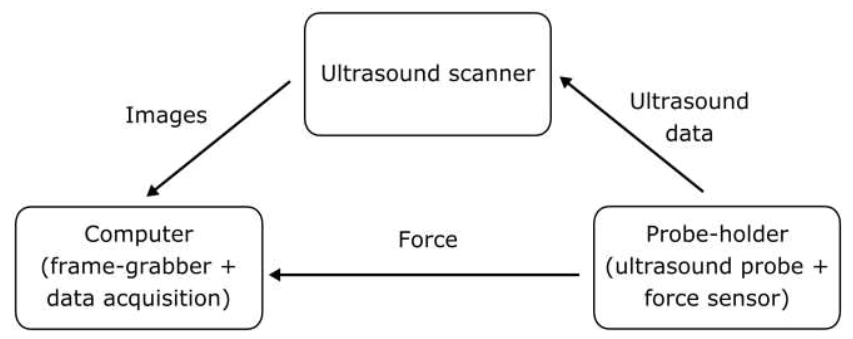

Fig. 2 Hardware architecture.

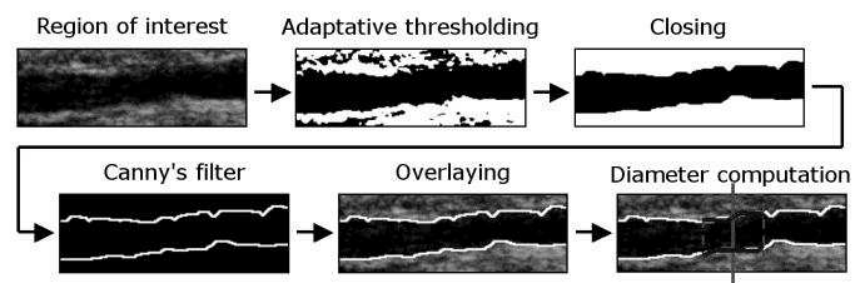

Fig. 3 The successive stages of image processing.

\section{B. Method}

Data acquisition was controlled by a laboratory-written piece of software synchronising force and video data acquisition and processing. One force value was obtained and saved for each echographic image. Another piece of software processed the acquired video sequence to extract the arterial diameter as follows:

First, image calibration was performed to allow pixel to millimetres conversion. Thereafter, the operator had to select the region of interest (ROI) in which the following analyses were performed (Fig.3):

- Closing operation: erosion followed by expansion allowing to remove artefacts and to smooth out the vessel's contour using a 9x9 structuring element on neighbourhood.

- Adaptive threshold: The suitable threshold level was calculated on the grey scale along a line parallel to the ultrasound beam,

- A Canny's filter [14], with a 3x3 window width, was applied.

- Contour superimposition was performed on each image.

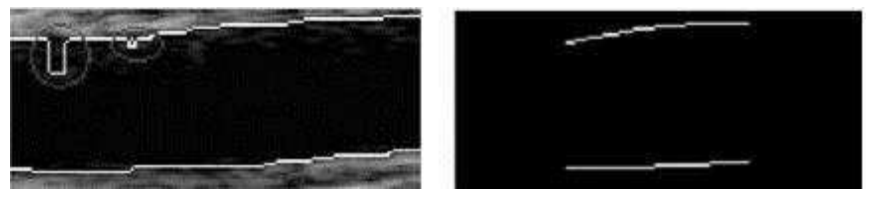

Fig. 4 Image processing with edges detection and smoothing by polynomial approximation.

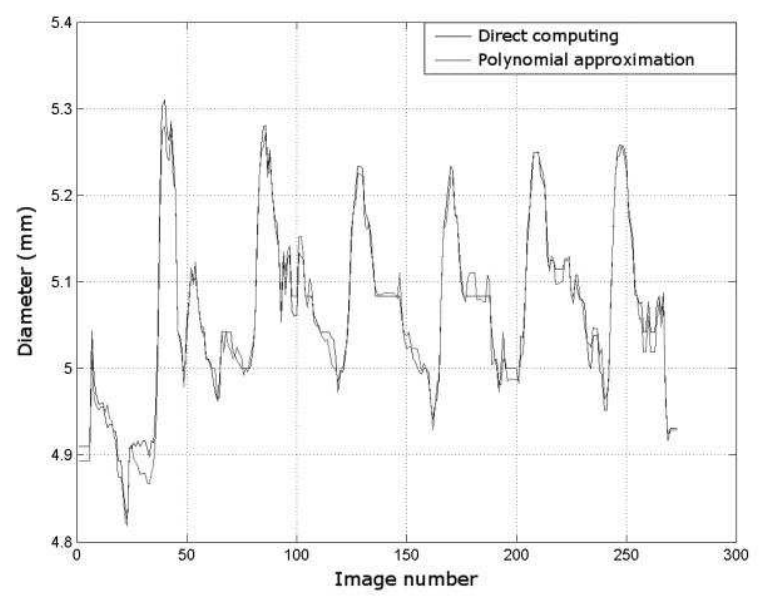

Fig. 5 Brachial artery diameter changes calculated on a sequence of images without and with polynomial approximation, in a normal subject.

At the centre of the ROI, a 20 pixels width area was automatically drawn, and the results of the previous stages of image processing were further processed for smoothing by $2^{\text {nd }}$-order polynomial approximation in order to be a part of the best-fit parabola following the arterial wall. This process enabled to suppress residual artefacts (Fig.4).

This 20 pixels width allowed eliminating the effect of isolated acoustic artefacts, thus overcoming the pitfall of other techniques like echo-tracking while avoiding the excessive temporal smoothing that would happen with a larger area because of the propagation of the arterial pulse wave (e.g. on the brachial artery of a normal subject, the pulse wave propagates along the wall with a velocity about 7 $\left.\mathrm{m} \cdot \mathrm{s}^{-1}\right)$.

The distance separating the two interpolated polynomials (i.e. proximal and far arterial wall) was then averaged to get the arterial diameter, and this calculation was repeated along the sequence of images (about 300 for a typical sequence). Fig. 5 shows the variation of the arterial diameter on a full sequence with a mean value of $5.06 \mathrm{~mm}$.

Frequency analysis of the resulting waveform yielded results a mode about of $1.2 \mathrm{~Hz}$, i.e. the normal frequency of heart beats. Direct calculation and polynomial approximation gave quite similar results because most artefacts had been already corrected by the operation of mathematical morphology. 


\section{Clinical Tests}

Clinical tests and measurements were performed in collaboration with vascular laboratory of the Nîmes University Hospital.:

- An Alpha 10 ProSound ultrasound scanner (HitachiAloka, Tokyo, Japan) with a $10 \mathrm{MHz}$ linear probe equipped with the above-mentioned force sensor.

- A Dinamap DPC320NR (GE Healthcare, Milwaukee, USA) electronic tensiometer.

- An articulated probe-holder mounted on a 3-axis adjustment device (MP-PH0001, Hitachi-Aloka, Tokyo, Japan).

- An ECG and breath-movement monitor with analog output.

When the sonographer handled the probe equipped with the force sensor without using the micrometric probe-holder for the examination of the brachial artery in normal volunteers, we were able to acquire and display simultaneously the force signal and the brachial artery diameter calculation results.

The results confirmed that arterial diameter measurements could be affected by the way the sonographer handled the probe, depending on the patient's size and posture, and that the force exerted on the probe could be quite different along the examination, and quite different between sonographers.

Therefore, the articulated probe-holder proved essential for accurate and reproducible measurements, allowing to maintain the ultrasound probe in the required position throughout the acquisition procedure, with a constant force. The probe-holder was used for all subsequent measurements.

\section{A. Relationship between arterial diameter and force:}

Fig.6 shows the effects of increasing the force applied by on the ultrasound probe on the brachial artery diameter. Increasing the force $\mathrm{F}$ from 0 to $6.75 \mathrm{~N}$ resulted in a decrease in mean arterial diameter $(\Phi)$ from 4.6 to $3 \mathrm{~mm}$ in this subject.

Performing a polynomial approximation of both the force and the arterial diameter curves, and subtracting the result from initial data, allowed us to centre the waveforms (Fig.7). We could then observe that with increasing of the force resulted in the diastole-to-systole diameter $(\Delta \Phi)$ and force feedback $(\Delta \mathrm{F})$ changes during the cardiac cycle increased jointly by a factor 2 , respectively from 0.15 to $0.3 \mathrm{~mm}$ and 0.175 to $0.350 \mathrm{~N}$. These observations supported our hypothesis that the force applied on the ultrasound probe during the examination does influence the measurement of arterial diameter, and the force feedback represents an image of the local blood pressure waveform.

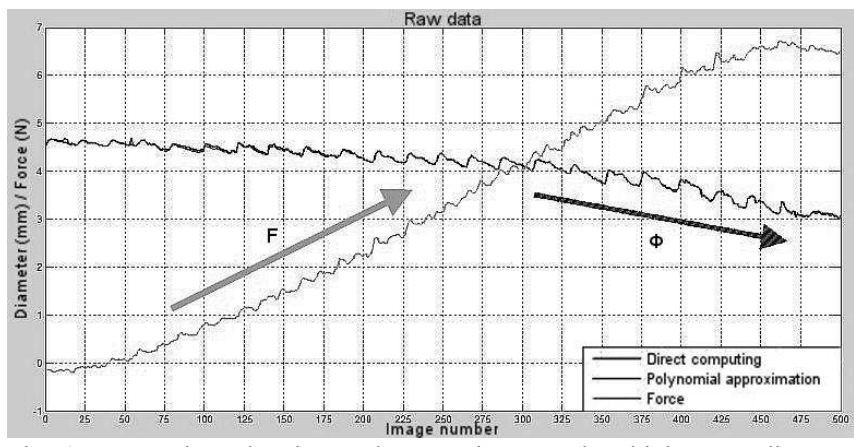

Fig. 6 Raw data showing a decrease in mean brachial artery diameter $(\Phi)$ when increasing the force $(\mathrm{F})$ exerted on the ultrasound probe.

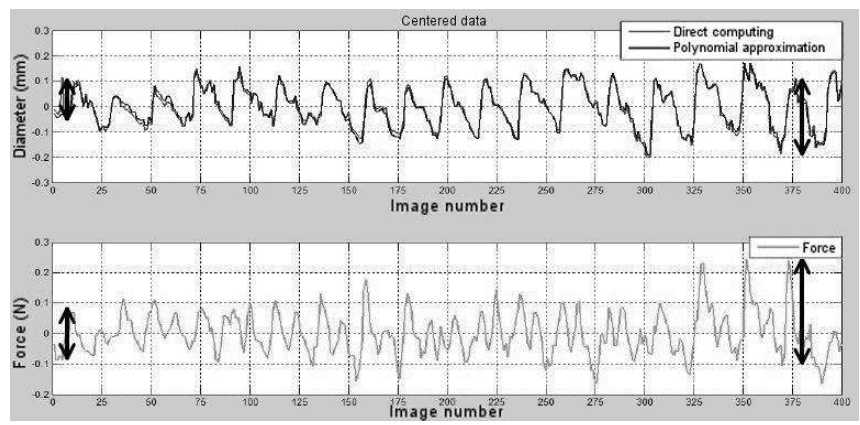

Fig. 7 Centred data showing an increase in diastole-to-systole brachial artery diameter changes when increasing the force exerted on the ultrasound probe.

\section{B. Interest of exerting a constant force:}

Using the probe-holder, we exerted a constant force on the ultrasound probe during a $20 \mathrm{~s}$ recording. This force was the minimal force allowing obtaining a stable and satisfying B-mode image of the brachial artery. The patient was first asked to breathe normally, then to maintain an apnoea. The first part of the graph showed 3 cycles of change in $\Delta \mathrm{F}$, whereas it remains stable during apnoea. The period of these cycles was roughly $4 \mathrm{~s}$, and corresponded to the respiratory rate. The diastole-to-systole diameter $(\Delta \Phi)$ and force feedback $(\Delta \mathrm{F})$ changes showed inverse variations during breathing (respectively $0.1 \mathrm{~mm}$ and $0.072 \mathrm{~N}$ ). This demonstrated that breathing affected diameter measurements probably by inducing slight arm movements..

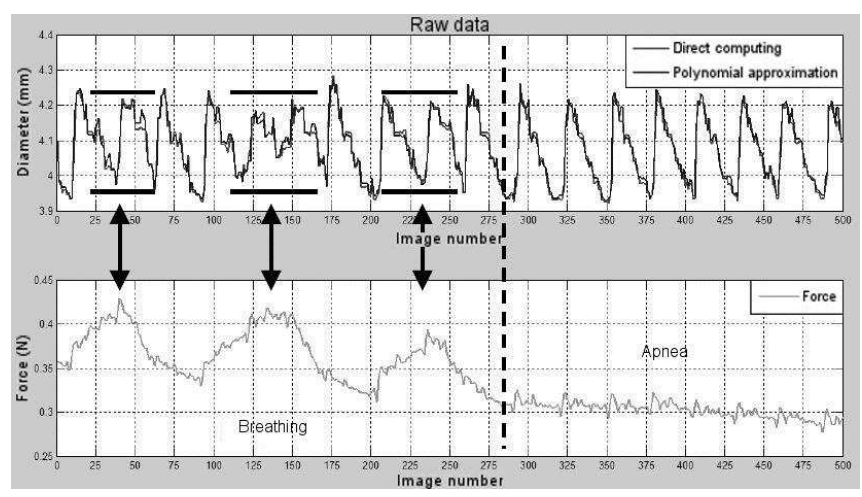

Fig. 8 Raw data showing brachial artery diastole-to-systole diameter and force feedback changes during breathing as measured with a constant force exerted on the ultrasound probe. 


\section{CONCLUSION}

The system we developed allowed simultaneous acquisition of ultrasound images and measurement of both the force exerted on the ultrasound probe by the sonographer and the force feedback resulting from arterial blood pressure pulsatility. We also developed image analysis software providing automatic and synchronized arterial diameter measurements. We were thus able to obtain measurements of the brachial artery diameter and diameter changes during the cardiac cycle, and showed the influence of the pressure exerted on the ultrasound probe by the operator on the arterial diameter and its cyclic variations. Our image analysis method has proved robust enough to correlate these force measurements with the arterial diameter and the amplitude of diameter changes during the cardiac cycle. We also demonstrated the ability of our system to capture the force feedbacks, providing an image of systemic blood pressure waveform. Moreover, we could show the influence of the breathing on arterial diameter changes.

Calibrating force feedback measurements would be needed before this approach can be used to offer a noninvasive method for providing the arterial blood pressure waveform. This can be achieved by simultaneous systemic blood pressure measurement by conventional method on the brachial artery on the other side, but this approach would have to be validated on a series of normal and pathologic subjects.

\section{REFERENCES}

[1] J. M. Bland and D. G. Altman, "Statistical Methods for Assessing Agreement Between Two Methods of Clinical Measurement," The Lancet, vol. 327, no. 8476, pp. 307-310, 1986.

[2] T. Gustavsson, R. Abu-Gharbieh, G. Hamarneh, and Q. Liang, "Implementation and Comparison of Four Different Boundary Detection Algorithms for Quantitative Ultrasonic Measurements of the Human Carotid Artery," in Cardiology 1997, vol. 24, no. December, pp. 69-72, 1997

[3] R. H. Selzer, W. J. Mack, P. L. Lee, H. Kwong-Fu, and H. N. Hodis, "Improved common carotid elasticity and intimamedia thickness measurements from computer analysis of sequential ultrasound frames," Atherosclerosis, vol. 154, no. 1, pp. 185-193, 2001.

[4] F. Beux, S. Carmassi, M. V. Salvetti, L. Ghiadoni, Y. Huang, S. Taddei, and A. Salvetti, "Automatic evaluation of arterial diameter variation from vascular echographic images," Ultrasound in Medicine \&amp; Biology, vol. 27, no. 12, pp. 1621-1629, 2001.

[5] B. Haluska, A. Sutherland, C. Case, R. Kennedy, and T. H. Marwick, "Automated edge-detection technique for measurement of brachial artery reactivity: a comparison of concordance with manual measurements," Ultrasound in Medicine \& Biology, vol. 27, no. 9, pp. 1285-1289, 2001.

[6] M. Cinthio, T. Jansson, A. R. Ahlgren, K. Lindström, and H. W. Persson, "A method for arterial diameter change measurements using ultrasonic B-mode data.," Ultrasound Med. Biol., vol. 36, no. 9, pp. 1504-12, Sep. 2010.

[7] T. Nilsson and S. Segstedt, "A robust and fast method for arterial lumen diameter and intima-media thickness measurements," IEEE Int. Ultrason. Symp. Proc., pp. 1678-1681, 2012.

[8] S. Graf, D. Craiem, and R. L. Armentano, "Non invasive assessment of carotid and femoral arterial pressure using B-mode ultrasound diameter waveforms.," Conf. Proc. IEEE Eng. Med. Biol. Soc., vol. 2012, pp. 5610-3, Jan. 2012.
[9] W. Yousheng, S. Xiaodi, and C. Jianxin, "A Method of Analyzing the Strain of Arterial Wall," 2009 Int. Forum Inf. Technol. Appl., no. 1, pp. 217-220, May 2009.

[10] F. Sun, Z. Liu, Y. Li, and P. Babyn, "Improved T-snake model based edge detection of the coronary arterial walls in intravascular ultrasound images," ICBBE, no. 1, pp. 1-4, 2009.

[11] N. Santhiyakumari, P. Rajendran, M. Madheswaran, and S. Suresh, "Detection of the intima and media layer thickness of ultrasound common carotid artery image using efficient active contour segmentation technique.," Med. Biol. Eng. Comput., vol. 49, no. 11, pp. 1299-310, Nov. 2011.

[12] J. Triboulet, E. Nasr, and M. Dauzat, "Evaluation of the Influence of Probe Pressure on the B-Mode Ultrasound Measurement of Arterial Diameter," in EMBC'06: $28^{\text {th }}$ Annual International Conference of the IEEE Engineering in Medicine and Biology Society. New York (USA): IEEE, 2006, pp. 3831-3835.

[13] J. Su, J.-J. Wang, C.-C. Chen, and L.-C. Lin, "A Cuff-Based Approach for Assessing the Flow-Mediated Dilatation of Brachial Arteries," 5th Int. Conf. Bioinforma. Biomed. Eng., no. 2, pp. 1-4, May 2011.

[14] J. F. Canny, "A Computational Approach to Edge Detection.” IEEE transactions on pattern analysis and machine intelligence, vol. 8, no. 6, pp. 679-98, Jun. 1986. 